\section{Avaliação antropométrica de pacientes com suspeita de erros inatos do metabolismo}

\section{Anthropometric evaluation of patients with suspected innate errors of metabolism}

Francilia de Kássia Brito-Silva 1

Ana Paula Pereira de Oliveira 2 Luiz Carlos Santana-da-Silva 3
1-3 Laboratório de Erros Inatos do Metabolismo. Instituto de Ciências Biológicas. Universidade Federal do Pará. Av. Augusto Corrêa, 01. Belém, PA, Brasil. CEP: 66.075-110. Email: lcss@ufpa.br

\begin{abstract}
Objectives: to provide an anthropometric evaluation of patients suspected of having innate errors of metabolism (IEMs) and report the prevalence of nutritional disorders (malnutrition, overweight and obesity)

Methods: fifty-five patients aged between 0 and 10 years were evaluated for anthropometric indices (H/A, W/A and W/H and BMI/A), in the innate errors of metabolism laboratory (LEIM) of the Federal University of Pará, using scales and an anthropometer. The data were collected using an LEIM form. Nutritional diagnosis was carried out using the Anthro and Anthro Plus programs and the SPSS statistics package.

Results: the patients attended were mostly aged between seven months and nine years. The main symptoms were delayed neuropsychomotor development and frequent infections. As for the nutritional status, a deficit of $23.7 \%$ was observed in weight for age, a deficit of $50.9 \%$ in height for age, and the prevalence of overweight and obesity was $15.4 \%$ according to weight for height, and 25,1\% according to body mass index for age.

Conclusions: the nutritional status of the patients was inadequate and, given the absence of a diagnosis of IEMs, the factors involved should be investigated more thoroughly.
\end{abstract}

Key words Anthropometry, Metabolism, inborn errors, Nutritional status

\section{Resumo}

Objetivos:avaliar antropometricamente os pacientes com suspeita de erros inatos do metabolismo (EIM) e descrever a prevalência de distúrbios nutricionais (desnutrição, sobrepeso e obesidade).

Métodos: foram avaliados 55 pacientes de 0 a 10 anos, de acordo com os índices antropométricos (A/I, P/I E P/A e IMC/I), no laboratório de erros inatos do metabolismo (LEIM) da Universidade Federal do Pará, através de balança e antropômetro. Os dados foram coletados a partir da ficha de atendimento do LEIM. Para o diagnóstico nutricional foram utilizados os programas Anthro e Anthro Plus e o programa SPSS para a análise estatística.

Resultados: os pacientes atendidos pertenciam, na maioria, a faixa etária de sete meses a nove anos. Os principais sintomas foram atraso no desenvolvimento neuropsicomotor e infecções frequentes. Quanto ao estado nutricional, foi observado déficit de 23,7\% no indicador de peso para idade, déficit de $50,9 \%$ no indicador de altura para idade, excesso de peso e obesidade de 15,4\% para peso para altura, e 25,1\% para índice de massa corporal para idade.

Conclusões: os pacientes apresentaram estado nutricional inadequados, o qual na ausência de diagnóstico de EIM, os fatores envolvidos devem ser mais bem averiguados.

Palavras-chave Antropometria, Erros inatos do metabolismo, Estado nutricional 


\section{Introdução}

Os erros inatos do metabolismo (EIM) são doenças hereditárias raras, transmitidas, geralmente, de forma autossômica recessiva, causadas por um defeito específico, normalmente enzimático, que consequentemente levará ao bloqueio de alguma via metabólica no organismo. Esse bloqueio por sua vez promove o acúmulo de substrato, ocasionando a diminuição do produto da reação ou o desvio do substrato para uma via metabólica alternativa. ${ }^{1}$

Os principais sintomas encontrados na fase neonatal são icterícia, acidose, convulsões e hipoglicemia. Recorrendo a história do paciente é possível identificar preferências ou aversões alimentares, odores, alterações tegumentares (dermatite seborréica), descompensação com doenças intercorrentes, dificuldades de ganho ponderal e vômitos recorrentes. No exame físico é possível identificar fácies grosseira, cabelos quebradiços, torcidos e com alopecia, pele com eritemas, distrofia ungueal e ictiose, movimentos oculares anormais, catarata e retinopatia, e hepatoesplenomegalia. $^{2}$

Além destes sintomas físicos encontra-se também, raquitismo, macro e microcefalia, perda de peso e desnutrição, distúrbios de crescimento, que vão desde déficit de crescimento estatural até crescimento exagerado para idade como na síndrome de marfan, que os indivíduos são altos e magros, ${ }^{3}$ além desta síndrome, exemplos de EIM que comprometem o desenvolvimento físico são mucopolissacaridose (MPS). 4

Pacientes com os sintomas descritos devem apresentar suspeita clínica de EIM. Para auxiliar no diagnóstico de muitos EIM que apresentam as características físicas supracitadas é necessário realizar avaliação antropométrica, que consiste num método de baixo custo e não invasivo. Este procedimento consiste em mensurar medidas corpóreas, como peso, altura, perímetro cefálico, pregas cutâneas, circunferências entre outros, a fim de calcular índices que indiquem o estado nutricional do individuo. 5

A antropometria é um método largamente utilizado em pesquisas e na clínica médica, logo se faz necessário dispor de parâmetros fidedignos. A Organização Mundial da Saúde (OMS) desenvolveu, para a avaliação antropométrica das crianças, novas curvas de crescimento para crianças de 0 a 5 anos em 2006 e de 5 a 19 anos em 2007, estas são uma referência do desenvolvimento infantil, as quais levaram em consideração o que seria um adequado desenvolvimento em termos de adequada alimentação, ausência de restrição econômica ou ambiental e potencial genético de crescimento.6,7

Como exemplo da aplicabilidade do método antropométrico pode-se citar estudos que buscam avaliar o estado nutricional. Como um estudo realizado na Bahia com recém-nascidos e utilizaram medidas de perímetro cefálico, distância biauricular, distância anteroposterior, tamanho da fontanela e índice cefálico, para definir medidas condizentes com a realidade da região, justificando-se pelo fato que as medidas antropométricas são um meio de detecção precoce de anomalias. ${ }^{8}$ Em São Paulo foi analisado o estado nutricional, através de avaliação antropométrica, de pré-escolares que frequentavam escolas municipais de educação infantil, encontrando dados que revelam sobrepeso e obesidade na população estudada. 9 Já em Brasília, DF, há prevalência de excesso de peso acima da média. 10 Em outro estudo11 realizado em Belém, PA, que teve como finalidade averiguar a prevalência de dislipidemia em escolares, através de um estudo transversal prospectivo, com 437 escolares, com idade entre 6 e 19 anos, dos quais foram obtidos medidas antropométricas de peso e altura para determinação do índice de massa corporal (IMC), encontrando $28,8 \%$ com sobrepeso e $36,2 \%$ com alto percentual de gordura corporal.

Considerando que a antropometria pode ser uma ferramenta útil como método colaborador ao diagnóstico e na ausência do mesmo, servirá para identificar o estado nutricional correlacionando-o com outros dados clínicos a fim de identificar outras possíveis causas dos sintomas físicos. Deste modo o presente estudo tem como objetivo avaliar antropometricamente crianças com suspeita de EIM, descrevendo a prevalência de distúrbios nutricionais (desnutrição, sobrepeso e obesidade) em crianças de 0 a 10, de acordo com os índices antropométricos (A/I, P/I E P/A e IMC/I). Discutir e comparar o estado nutricional de acordo com a sintomatologia apresentado pelos pacientes, atraso do desenvolvimento neuropsicomotor (ADNPM), regressão neuropsicomotora (RNPM), infecções frequentes, problemas intestinais, duração do aleitamento materno, inicio dos sintomas, dado-socioeconômicos e de consanguinidade. Com a perspectiva de averiguar o estado nutricional e clínico dessas crianças, de modo a instigar novas pesquisas, que possam aprofundar a correlação do estado nutricional com os sintomas clínicos e com o próprio EIM quando este estiver presente. 


\section{Métodos}

Estudo transversal com crianças que apresentam suspeita de EIM encaminhados ao Laboratório de Erros Inatos do Metabolismo (LEIM) da Universidade Federal do Pará (UFPA).

A amostra é representativa da população de pacientes atendidos no LEIM, visto que envolveu 55 crianças na faixa etária de 0 a 10 anos de idade, que foram atendidos no período de maio de 2010 a janeiro de 2011. Neste mesmo período o LEIM atendeu 170 pacientes, destes 133 pertenciam à faixa etária de 0 a 10 anos. O restante não pôde participar por vários motivos: responsável não levou a criança no dia da entrevista, inviabilidade de deslocamento da criança da cidade de origem, internação hospitalar, não aceitação em participar da pesquisa, dentre outros motivos.

Os pacientes com suspeita de EIM foram encaminhados pela rede pública e privada de saúde ao LEIM da UFPA, oriundos de Belém e de outros municípios do Estado do Pará e da Região Norte. Foram excluídos do estudo os pacientes maiores de dez anos, e aqueles que não puderam por algum motivo participar desse estudo.

Utilizou-se para coleta dos dados a ficha de atendimento do LEIM da UFPA, contendo dados clínicos, socioeconômicos e de peso e altura atual, aferidos no momento da entrevista.

$\mathrm{O}$ peso e altura atual foram aferidos segundo o procedimento do Sistema de Vigilância Alimentar Nutricional (SISVAN): orientações básicas para a coleta, processamento, análise de dados e informação em serviços de saúde. 12

Utilizou-se para crianças menores de dois anos, balança digital mobile baby da Balmak capacidade máxima de $25 \mathrm{~kg}$ e mínima de $40 \mathrm{~g}, \mathrm{e}=2 \mathrm{~g} / 5 \mathrm{~g}$, e infantômetro com capacidade de um metro e três centímetros. Em casos de impossibilidade de a criança ser avaliada, como nos casos de alterações graves neuropsicomotoras ou de outros sintomas clínicos ou de estresse em que impedissem a avaliação isolada das crianças, o procedimento adotado foi a aferição do peso da criança junto com a sua mãe (ou responsável). Posteriormente, o peso da criança foi corrigido mediante subtração do peso da mãe.

Para as crianças maiores de dois anos a altura foi aferida com utilização de estadiômetro portátil da marca Alturaexata com capacidade de 2,13 metros e resolução de $1 \mathrm{~mm}$. O peso foi aferido com o uso de uma balança eletrônica (G-Tech) com capacidade de ate 150 quilos e precisão de 100 gramas.

A análise dos dados foi realizada através de duas formas. No primeiro momento o estado nutricional, obtido por antropometria, foi avaliado de acordo com o gênero e faixa etária, através dos indicadores de peso para idade, altura para idade, peso para altura e IMC para idade, no programa Anthro e Anthro plus, que utiliza os padrões da World Health Organization (WHO) em escore Z. ${ }^{13}$ No segundo momento, as análises ocorreram no programa Statistical Package for Social Sciences - SPSS, utilizando análises de frequência simples.

Quanto aos aspectos éticos este trabalho foi aprovado pelo Comitê de Ética em Pesquisa do Hospital Universitário João de Barros Barreto, conforme resolução 196/96 do Conselho Nacional de Saúde (submetido em 11/01/2010, protocolo $\mathrm{n}^{\circ}$ $048 / 2010$, e aprovado na reunião do dia 25/05/2010). Foi utilizado termo de consentimento livre e esclarecido e carta convite, para que as crianças participassem da pesquisa.

\section{Resultados}

A Tabela 1 mostra a distribuição em porcentagem das características da família e dos pacientes com suspeita de EIM. Não houve diferença estatística entre os gêneros masculino e feminino. A faixa etária mais encontrada esteve entre sete meses e abaixo de nove anos $(85,5 \%)$. Os pacientes com suspeita de EIM foram enviados especialmente pelos serviços públicos de saúde $(87,3 \%)$. Mais da metade das famílias apresenta renda salarial menor que dois salários mínimos por mês $(54,6 \%)$. Quanto à escolaridade, foi observado que quase a metade dos pais $(43,6 \%)$ não completou o primeiro grau. A taxa de consanguinidade foi elevada $(23,6 \%)$.

Em relação ao indicador de peso para idade foi observado que $70,9 \%$ (39/55) das crianças estavam eutróficas ou sem desnutrição aguda. No entanto, $23,7 \%(13 / 55)$ se encontravam com peso abaixo do recomendado para sua idade, caracterizando um quadro grave de desnutrição nestas crianças. Quanto ao indicador de altura para idade relacionada à presença de desnutrição crônica, mais da metade dos pacientes com suspeita de EIM apresentaram baixa estatura recomendada para idade $(50,9 \%$ ou $28 / 55)$, caracterizando o atraso no crescimento destas crianças. Para o indicador de peso para altura em crianças de zero a cinco anos e IMC/I em crianças entre cinco e dez anos, os resultados mostraram que mesmo a maioria se encontrando com indicadores adequados $(61,5 \%$ e $68,8 \%$ respectivamente), vale ressaltar que $15,4 \%$ e $25,1 \%$ destas crianças já apresentavam sobrepeso e obesidade em relação aos indicadores recomendados de peso para sua altura e em 
relação ao IMC para idade, respectivamente (Tabela 2). A frequência encontrada para as crianças que fizeram aleitamento materno foi de 87,3\%. Em relação aos sinais e sintomas apresentados pelos pacientes como motivo do encaminhamento ao LEIM foi verificado que o início dos sintomas ocorre antes das crianças completarem dois anos $(81,8 \%)$. Sessenta por cento da amostra (33/55) apresentavam atraso no desenvolvimento neuropsicomotor e $61,8 \%$ também relatavam infecções frequentes como pneumonia, infecções no trato urinário e intestinal, com destaque para $25,5 \%$ delas também apresentarem

Tabela 1

Distribuição percentual das características sócio demográficas da família e dos pacientes com suspeita de erros inatos do metabolismo, Belém, 2011.

\begin{tabular}{|c|c|c|}
\hline Variável & $\mathbf{N}$ & $\%$ \\
\hline \multicolumn{3}{|l|}{ Gênero } \\
\hline Masculino & 30 & 54,5 \\
\hline Feminino & 25 & 45,5 \\
\hline \multicolumn{3}{|l|}{ Faixa etária } \\
\hline 0 a 6 meses & 5 & 9,1 \\
\hline 7 meses a 1 ano e 11 meses & 16 & 29,1 \\
\hline 2 a 2 anos e 11 meses & 9 & 16,4 \\
\hline 3 a 5 anos e 11 meses & 11 & 20,0 \\
\hline 6 a 8 anos e 11 meses & 11 & 20,0 \\
\hline 9 a 10 anos & 3 & 5,5 \\
\hline \multicolumn{3}{|l|}{ Serviço requisitante } \\
\hline SUS & 48 & 87,3 \\
\hline Privado & 7 & 12,7 \\
\hline \multicolumn{3}{|l|}{ Renda (salário mínimo) } \\
\hline$<1$ & 10 & 18,2 \\
\hline 1,5 & 20 & 36,4 \\
\hline 3 & 12 & 21,8 \\
\hline 8 & 4 & 7,3 \\
\hline$>8$ & 2 & 3,6 \\
\hline Sem renda & 3 & 5,5 \\
\hline \multicolumn{3}{|l|}{ Escolaridade da mãe } \\
\hline Analfabeto & 0 & - \\
\hline $1^{\circ}$ grau completo & 3 & 5,5 \\
\hline $1^{\circ} \mathrm{grau}$ incompleto & 24 & 43,6 \\
\hline $2^{\circ}$ grau completo & 17 & 30,9 \\
\hline $2^{\circ} \mathrm{grau}$ incompleto & 3 & 5,5 \\
\hline Superior & 6 & 10,9 \\
\hline Não sabe & 2 & 3,6 \\
\hline \multicolumn{3}{|l|}{ Escolaridade do pai } \\
\hline Analfabeto & 2 & 3,6 \\
\hline $1^{\circ}$ grau completo & 2 & 3,6 \\
\hline $1^{\circ} \mathrm{grau}$ incompleto & 24 & 43,6 \\
\hline $2^{\circ}$ grau completo & 11 & 20 \\
\hline $2^{\circ} \mathrm{grau}$ incompleto & 3 & 5,5 \\
\hline Superior & 6 & 10,9 \\
\hline Não sabe & 7 & 12,7 \\
\hline \multicolumn{3}{|l|}{ Pais consanguíneos } \\
\hline Não & 41 & 74,5 \\
\hline Sim & 13 & 23,6 \\
\hline Não sabe & 1 & 1,8 \\
\hline
\end{tabular}


problemas intestinais apesar da maioria não relatar casos como diarreia e constipação. A convulsão também esteve presente em $40 \%$ dos casos (Tabela $3)$.

No que se refere a diagnóstico de EIM, dentre os 55 pacientes participantes da pesquisa, dois destes foram diagnosticados com EIM (um com mucopolissacaridose e outro com Doença de Sandhoff).

Os diagnósticos foram realizados por meio de uma parceria entre o LEIM da UFPA e o Serviço de Genética Médica do Hospital de Clínicas de Porto Alegre (SGM-HCPA).

O paciente com MPS IIIB é do gênero masculino. $\mathrm{Na}$ época do estudo apresentava sete anos e três meses. Foi encaminhado ao LEIM por apresentar ADNPM, retardo mental, e infecções frequentes. Os sintomas iniciaram aos quatro anos de vida.

Quanto ao estado nutricional, o paciente com MPS apresenta P/I adequado, assim como a A/I. No entanto, apresenta sobrepeso no indicador IMC para Idade.

A paciente com doença de Sandhoff pertence ao gênero feminino e na época do estudo contava com dois anos e três meses. Os pais são consanguíneos e os sintomas iniciaram aos nove meses de vida. Quanto ao estado nutricional, a paciente apresentou $\mathrm{P} / \mathrm{I}$ e $\mathrm{P} / \mathrm{A}$ adequados e, baixa estatura para idade. Este último indicador pode estar relacionado com a deficiência no metabolismo da paciente que pode estar ocasionando um atraso no crescimento.

\section{Tabela 2}

Distribuição percentual do estado nutricional de pacientes com suspeita de erros inatos de metabolismo. Belém, PA, 2011.

\begin{tabular}{|c|c|c|}
\hline Variável & $\mathbf{N}$ & $\%$ \\
\hline \multicolumn{3}{|l|}{ Peso para idade } \\
\hline Muito baixo peso/idade & 4 & 7,3 \\
\hline Baixo peso/idade & 9 & 16,4 \\
\hline Peso adequado & 39 & 70,9 \\
\hline Peso elevado /idade & 3 & 5,5 \\
\hline \multicolumn{3}{|l|}{ Altura para idade } \\
\hline Muito baixa $A / I$ & 18 & 32,7 \\
\hline Baixa A/I & 10 & 18,2 \\
\hline Adequada $A / I$ & 27 & 49,1 \\
\hline \multicolumn{3}{|l|}{ Peso para altura* } \\
\hline Magreza acentuada & 1 & 2,6 \\
\hline Magreza & 1 & 2,6 \\
\hline Eutrofia & 24 & 61,5 \\
\hline Risco de sobrepeso & 7 & 17,9 \\
\hline Sobrepeso & 3 & 7,7 \\
\hline Obesidade & 3 & 7,7 \\
\hline \multicolumn{3}{|c|}{ Índice de massa corporal para idade** } \\
\hline Magreza & 1 & 6,3 \\
\hline Eutrofia & 11 & 68,8 \\
\hline Sobrepeso & 3 & 18,8 \\
\hline Obesidade & 1 & 6,3 \\
\hline
\end{tabular}

*O escore de peso para altura é apenas para crianças de 0 a 5 anos. Nessa variável o $\mathrm{n}=39$ pacientes; **O escore de índice de massa corporal para idade é apenas para crianças de 5 a 10 anos. Nessa variável o $n=16$ pacientes. 
Perfil de aleitamento materno e distribuição percentual dos sinais e sintomas de pacientes com suspeita de erros inatos do metabolismo. Belém, PA, 2011.

\begin{tabular}{|c|c|c|}
\hline Variável & $\mathbf{N}$ & $\%$ \\
\hline \multicolumn{3}{|c|}{ Duração do aleitamento materno } \\
\hline Não mamou & 5 & 9,1 \\
\hline$<1$ mês & 4 & 7,3 \\
\hline$>1$ a 6 meses & 7 & 12,7 \\
\hline$>6$ meses a 1 ano & 7 & 12,7 \\
\hline$>1$ ano & 16 & 29,1 \\
\hline Ainda mama & 14 & 25,5 \\
\hline Não sabe & 2 & 3,6 \\
\hline ADNPM* & 33 & 60,0 \\
\hline RNPM** & 4 & 7,3 \\
\hline Infecções frequentes & 34 & 61,8 \\
\hline Convulsão & 22 & 40,0 \\
\hline Problemas intestinais & 14 & 25,5 \\
\hline \multicolumn{3}{|l|}{ Inicio dos sintomas } \\
\hline 0 a 6 meses & 27 & 49,1 \\
\hline 7 meses a 1 ano e 11 meses & 18 & 32,7 \\
\hline 2 a 2 anos e 11 meses & 4 & 7,3 \\
\hline 3 a 5 anos e 11 meses & 5 & 9,1 \\
\hline 6 a 8 anos e 11 meses & 1 & 1,8 \\
\hline
\end{tabular}

*ADNPM= atraso no desenvolvimento neuropsicomotor; **RNPM= regressão neuropsicomotora.

\section{Discussão}

A média de faixa etária dos pacientes com suspeita de apresentar EIM foi de três anos, e uma frequência de $56 \%$ para a faixa etária compreendida entre dois anos a oito anos e 11 meses. Este achado se aproxima do encontrado na literatura, o qual a média da faixa etária foi entre quatro anos e oito meses $14 \mathrm{e}$ $30 \%$ de pacientes com suspeita de apresentam EIM em uma faixa etária compreendida entre dois a seis anos. 15

Já no que se refere a renda familiar, segundo dados do Ministério da Saúde, a renda média domiciliar per capita na Região Norte é R\$ 580,19 reais e no Pará é de $\mathrm{R} \$ 415,05$ reais, dos indivíduos atendidos pelo Sistema Único de Saúde (SUS). Em relação à amostra estudada, cerca de $36,0 \%$ recebe entre 1 a 1,5 salários mínimos, o que está de acordo com a renda média domiciliar per capita da Região Norte do Brasil. 16

Outro item é a consanguinidade, entre os pais é um ponto importante a ser observado quando na suspeita de EIM. Estudos demonstram uma frequência de consanguinidade em torno de $6,6 \% 15 \mathrm{e}$ de 7,6\%.17 As frequências encontradas para casamento consanguíneo no presente estudo foram de
23,6\%. Esta frequência pode estar superestimada, considerando que o tamanho amostral nos trabalhos anteriores foi expressivamente maior.

Não há relatos na literatura especializada sobre o estado nutricional de pacientes com suspeita de EIM, por este motivo foram utilizados dados de pesquisa com crianças que não tinham suspeita de EIM, os quais foram úteis para comparar e demonstrar se nossos pacientes de fato, por apresentarem suspeita EIM ou na presença de diagnóstico, no que se refere ao estado nutricional, estão apresentando distúrbios nutricionais leve, acentuado ou grave.

Uma pesquisa de âmbito nacional demonstrou que o indicador de peso para idade $(\mathrm{P} / \mathrm{I})$ no Brasil revela $1,8 \%$ dos indivíduos com déficit de peso para idade, $1,5 \%$ para peso para altura $(\mathrm{P} / \mathrm{A}), 6,7 \%$ de altura para idade (A/I) e excesso de peso para altura $7 \%$. Sendo que na Região Norte foi encontrado déficit de A/I, P/A e P/I de $14,7 \%, 0,5 \%$ e 3,3\%, respectivamente, e excesso de peso para altura de $6,2 \% .{ }^{18}$ Os indicadores desses dados nacionais e o da Região Norte, encontram-se melhores do que o encontrado no presente estudo, nos quais os indicadores demonstraram déficit de $\mathrm{P} / \mathrm{I}$ cerca de 13 vezes maior, déficit de A/I em média cinco vezes maior, e a soma de excesso de peso e obesidade para 
P/A (crianças de zero a cinco anos) foi duas vezes maior. Estes dados permitem inferir que os EIM são doenças que podem interferir no estado nutricional dos indivíduos, e na ausência do diagnóstico, como o que ocorre com a maioria dos pacientes da amostra, cabe investigar os fatores associados ao grande percentual de inadequação de estado nutricional, uma vez que por serem doenças raras, dos 55 pacientes participantes, uma minoria teve diagnóstico de EIM.

Dentre os possíveis fatores associados, pode-se inferir a renda. Pois na variável renda a maioria dos participantes referiu ter renda inferior a 1,5 salários mínimos. Sob este aspecto os nossos dados podem estar de acordo com o encontrado em uma pesquisa nacional, 19 que demonstrou que a renda está intimamente ligada ao estado nutricional. O estudo demonstrou que o déficit de altura esteve presente em crianças cujas rendas familiares foram menores que um quarto de salário mínimo per capita. Assim como o déficit de peso, mas apenas para os meninos. No entanto, para ambos os gêneros o excesso de peso e obesidade aumentou com aumento da renda. 19

Outro fator que pode estar associado com os distúrbios nutricionais encontrados, é o aleitamento materno. O Ministério da Saúde (MS) do Brasil e a Organização Mundial da Saúde (OMS) preconizam que a duração do aleitamento materno seja ate os dois anos de vida ou mais, sendo o aleitamento materno exclusivo nos primeiros seis meses de vida. 20 Do total de indivíduos estudados menores de dois anos (22 crianças) que deveriam estar em aleitamento materno, cerca de $40 \%$ não se encontravam mais nessa condição.

A II Pesquisa de Prevalência de Aleitamento Materno nas Capitais Brasileiras e Distrito Federal, realizada pelo MS apontaram que o aleitamento materno exclusivo em menores de seis meses na região norte foi de $45,9 \%$, em Belém de $56,1 \%$ e no Brasil 58,74\%.21 Apesar de a OMS preconizar que o aleitamento materno deve ser até dois anos, no Brasil ele está entre 10 e 20 meses, ou seja, em uma faixa inadequada. No presente trabalho $87,3 \%$ dos participantes iniciaram o aleitamento materno, isto sem levar em consideração a duração. Dado que se aproxima ao encontrado na literatura $95 \% .18,22 \mathrm{Na}$ maioria dos casos de EIM os sintomas se manifestam no primeiro ano de vida, ${ }^{23}$ fato este observado no presente trabalho. E as principais manifestações clínicas em pacientes com EIM, são a convulsão, atraso no desenvolvimento físico e psicomotor e infecção em crianças até os dez anos. 23 Em relação às convulsões foi encontrada uma frequência de $40 \%$, abaixo das frequências de $58,0 \% 15$ e $59,4 \%, 17$ encontrado em outros trabalhos.

$\mathrm{Na}$ literatura tem-se relato de frequência de infecção $29,4 \%, 17$ valor abaixo do encontrado no presente estudo, que foi de $61,8 \%$. A infecção está associada às causas de estresse metabólico agudo na infância. No entanto os EIM podem também ser uma causa primária para a infecção.

A frequência de EIM encontrada no presente estudo, que foi de 3,6\% (2/55) é menor do que a de $7,2 \% 15$ e, similar à de $3 \% 24$ e maior do que $1,98 \%, 14$ encontrado em estudos sobre EIM.

Dos dois pacientes de EIM deste trabalho, um é o caso de MPS do tipo IIIB, a qual é causada pela deficiência da enzima Alfa-N-acetilglicosaminidase, culminando com o acúmulo nos lisossomos de glicosaminoglicanos. Esta forma de MPS apresenta um padrão de herança autossômica recessiva. Clinicamente a MPS IIIB é caracterizada pelo profundo retardo mental e pelo relativo envolvimento visceral e esquelético. 25 Este paciente apresentou adequação no indicador A/I, desta forma, segundo este indicador confirma-se que o comprometimento ósseo é relativo como descrito na literaura. 25 Com relação ao sobrepeso no indicador IMC para idade, pode-se citar um estudo realizado em Belém-PA, onde um paciente com MPS tipo I apresentou sobrepeso (dados do LEIM, ainda não publicados). O segundo caso de EIM trata-se da Doença de Sandhoff, uma esfingolipidose. Esta é uma desordem neurodegenerativa progressiva, caracterizada pelo acumulo de gangliosidio GM2, por deficiência das enzimas: hexosaminidase A (Hex A) e hexosaminidase B (Hex B). 26 Os pacientes apresentam macrocefalia, neurodegeneração, regressão psicomotora, tetraparesia espática, descerebração, mancha vermelho-cereja (forma infantil), algumas vezes retinopatia (forma juvenil) e hepatoesplenomegalia. 27

O caso da Doença de Sandhoff encontrado neste trabalho apresenta quadro clínico semelhante ao apresentado em um relato de caso sobre esta doença, no qual descreveu um paciente do gênero masculino (um ano e seis meses), que também apresentava regressão psicomotora e a mancha vermelho-cereja na retina, inicio dos sintomas aos seis meses de vida e pais consanguíneos (primos de primeiro grau). 28

Foi possível concluir neste trabalho, que o EIM repercute negativamente no estado nutricional dos pacientes, uma vez que as duas crianças que tiveram diagnóstico apresentaram algum distúrbio nutricional.

Dentre os pacientes que não tiveram diagnóstico de EIM, um percentual significativo também apresentou estado nutricional inadequado, segundo os 
indicadores de A/I, P/A, P/I e IMC/I. Ressaltando por exemplo déficit de $23,7 \%$ no indicador de peso para idade, déficit de $50,9 \%$ no indicador de altura para idade, excesso de peso e obesidade de 15,4\% para peso para altura, e $25,1 \%$ para IMC para idade. Percentuais que confirmam a hipótese que os pacientes com suspeita de EIM, apresentam problemas nutricionais, que não estão ligados necessariamente a doenças metabólicas, e sim mascarando uma falsa suspeita para estas doenças. Essa suspeita pode surgir por envolvimento de fatores socioeconômicos ou de aleitamento materno, ou outros que não foram aqui discutidos, que por sua vez podem estar levando a sinais e sintomas típicos de pacientes com EIM, que por sua vez podem estar relacionados com os distúrbios nutricionais, ou vice-versa.

Muitas hipóteses podem ser levantadas a partir do encontrado neste trabalho, e dar margem para novas investigações, que possam elucidar a verdadeira causa destes sintomas e do inadequado estado nutricional encontrado nos pacientes com suspeita de EIM.

\section{Referências}

1. Karam SM, Schwartz IVD, Giugliani R. Introdução e aspectos clínicos. In: Carakushansky G, editor. Doenças genéticas em pediatria. Rio de Janeiro: Guanabara-Koogan; 2001. p. 155-8.

2. Araújo APQC. Doenças metabólicas com manifestações psiquiátricas. Rev Psiq Clin. 2004; 31: 285-9.

3. Martins AM, Frangipani BJ, Micheletti C, Oliveira RBO Protocolo Brasileiro de Dietas: erros inatos do metabolismo. São Paulo: Segmento Farma; 2006.

4. Martins AM, Dualibi AP, Norato DN, Takata ET, Santos ES, Valadares ER, Porta G, de Luca G, Moreira G,Pimentel H, Coelho J, Brum JM, Semionato Filho J, Kerstenetzky MS, Guimarães MR, Rojas MV, Aranda PC, Pires RF, Faria RG, Mota RM, Matte U, Guedes ZC. Guidelines for the management of mucopolysaccharidosis Type I. J Pediatrics. 2009; 155 (Suppl. 2): 32-46.

5. Flores-Huerta S. Antropometría, estado nutricio y salud de los niños: importancia de las mediciones comparables. Bol Med Hosp Infant Mex. 2006; 63: 73-5.

6. Victora CG, Araújo CL, Onis M. Uma nova curva de crescimento para o século XXI. Acessado: http://nutricao.saude. gov.br/sisvan.php?conteudo=curvas_cresc_oms [acesso em 3 set 2009].

7. Brasil. SISVAN- Curvas de crescimento da Organização Mundial da Saúde-OMS. http://nutricao.saude.gov.br/ sisvan.php?conteudo=curvas_cresc_oms. Acessado: 03/09/09.

8. Mota M, Melo A, Burak C, Daltro C, Rodrigues B, Lucena R. Antropométrica craniana de recém-nascidos normais Arq Neuropsiquiatr. 2004; 62: 626-9.

9. Fernades IT, Gallo RP, Advíncula AO. Avaliação antropométrica de pré-escolares do município de Mogi-
A antropometria pode ser um método colaborador no diagnóstico de EIM, uma vez que os pacientes que apresentam este tipo de doença apresentam distúrbios nutricionais que podem ser diagnosticados através deste método de avaliação.

Deste modo na ausência do diagnóstico de EIM de um paciente que a princípio apresentou a suspeita de EIM, a conduta pertinente a ser adotada é a investigação de outros fatores envolvidos na etiologia do quadro clínico e nutricional do paciente. A renda, os parâmetros antropométricos, aleitamento materno, sinais e sintomas devem ser analisados em conjunto para investigar ou caracterizar as manifestações clínicas apresentados pelos pacientes e assim definir o diagnóstico de outras doenças que envolvem tais variáveis, incluindo o estado nutricional.

Portanto, é importante dar continuidade ao trabalho relacionando os possíveis fatores causadores do inadequado estado nutricional, assim como averiguação dos hábitos alimentares e orientação dos responsáveis quanto à alimentação adequada e de baixo custo e aleitamento materno.

Guaçu, São Paulo: subsidio para políticas públicas de saúde. Rev Bras Saúde Matern Infant. 2006; 6: 217-22.

10. Tuma-Brito RCF, Costa THM, Schmitz BAS. Avaliação antropométrica e dietética de pré-escolares em três creches de Brasília, Distrito Federal. Rev Bras Saúde Matern Infant. 2005; 5: 419-28.

11. Ribas AS, Santana-da-Silva, LC. Dyslipidemia in schoolchildren from private schools in Belém. Arq Bras Cardiol. 2009; 92: 412-7.

12. Brasil. Ministério da Saúde. Vigilância Alimentar e Nutricional (SISVAN). Orientações para a coleta, o processamento, a analise de dados e informações em serviços de saúde. Brasília, DF; 2004.

13. WHO (World Health Organization). Curvas de crescimento 2006 e 2007. [acesso em 4 set 2009]. Disponível em: http://www.who.int/childgrowth/en

14. Amancio FAM, Scalco FB, Coelho CB. Investigação diagnostica de erros inatos do metabolismo em um hospital universitário. J Bras Patol Med Lab. 2007; 43: 169-74.

15. Simoni RE, Oliveira CPH, Grassiano DM Santos CMRC, Baruque MGA, Gomes LNF, ET AL. Screening for inborn errors of metabolism in highrisk children from Rio de Janeiro, Brazil. Early Hum Dev. 1998; 50: 305-11.

16. Brasil. Ministério da Saúde. DATA SUS. [acesso em 20 mai 2011]. Disponível em: http://tabnet.datasus.gov.br/cgi/ idb2009/b08uf tot.htm

17. Souza ICN, Martins AM, Almeida V, Santana-da-Silva LC. Triagem urinária para erros inatos do metabolismo em crianças com atraso no desenvolvimento. Rev Para Med. 2007; 21: 23-8

18. Brasil. Ministério da Saúde. Centro Brasileiro de Análise e Planejamento. Pesquisa Nacional de Demografia e Saúde 
da Criança e da Mulher - PNDS 2006: dimensões do processo reprodutivo e da saúde da criança. Brasília, DF; 2009 .

19. Brasil. POF (Pesquisa de orçamentos Familiares 2008 2009). Antropometria e estado nutricional de crianças, adolescentes e adultos no Brasil. Rio de janeiro: IBGE; 2010

20. Brasil. Ministério da Saúde. Secretaria de Políticas de Saúde. Organização Pan Americana da Saúde. Guia alimentar para crianças menores de dois anos. Brasília, DF; 2002.

21. Brasil. Ministério da Saúde. Secretaria de Atenção a Saúde. Departamento de Ações Programáticas e Estratégicas. II Pesquisa de prevalência de aleitamento materno nas capitais brasileiras e Distrito Federal. Brasília, DF; 2009. 108 p

22. Sousa N, Bernardes AC. Aleitamento materno: prevalência e caracterização da informação prestada. Rev Port Clin Geral. 2010; 26: 440-8.

23. Wajner MCRV, Burin M, Giugliani R, Coelho JC. Investigação de erros inatos do metabolismo. Rev HCPA 2001; $1: 343-60$

Recebido em 31 de agosto de 2011

Versão final apresentada em 3 de julho de 2012

Aprovado em 15 de agosto de 2012
24. Oliveira AC, Nunes MAS, Martins, AM, Almeida V. Screening for inborn errors of metabolism among newborns with metabolic disturbance and/or neurological manifestations without determined cause. Rev Paul Med. 2001; 119: $160-4$.

25. Scriver CR, Beaudet AL, Sly WS, Valle D. The metabolic and molecular basis of inherited disease. 8 ed. New York: McGraw-Hill, Inc; 2001.

26. Mahuran DJ. Biochemical consequences of mutations causing the GM2 gangliosidoses. Biochim Biophys Acta. 1999; 1455: 105-38.

27. Heidelberg JZ, Heidelberg GFH. Valdemecum metabolicum: manual de pediatria metabólica. 2 ed. Alemanha: Segmento farma; 2004

28. Saouab R, Mahi M, Abilkacem R, Boumdin H, Chaouir S, Agader O, Amil T, Hanine A. A case report of Sandhoff Disease. Clin Neuroradiol. 2010; 21: 83-5. 